\title{
Mining and analysis of audiology data to find significant factors associated with tinnitus masker
}

\author{
Muhammad Naveed Anwar
}

\begin{abstract}
Objectives: The objective of this research is to find the factors associated with tinnitus masker from the literature, and by using the large amount of audiology data available from a large NHS (National Health Services, UK) hearing aid clinic. The factors evaluated were hearing impairment, age, gender, hearing aid type, mould and clinical comments.
\end{abstract}

Design: The research includes literature survey for factors associated with tinnitus masker, and performs the analysis of audiology data using statistical and data mining techniques.

Setting: This research uses a large audiology data but it also faced the problem of limited data for tinnitus.

Participants: It uses 1,316 records for tinnitus and other diagnoses, and 10,437 records of clinical comments from a hearing aid clinic.

Primary and secondary outcome measures: The research is looking for variables associated with tinnitus masker, and in future, these variables can be combined into a single model to develop a decision support system to predict about tinnitus masker for a patient.

Results: The results demonstrated that tinnitus maskers are more likely to be fit to individuals with milder forms of hearing loss, and the factors age, gender, type of hearing aid and mould were all found significantly associated with tinnitus masker. In particular, those patients having Age $<=55$ years were more likely to wear a tinnitus masker, as well as those with milder forms of hearing loss. ITE (in the ear) hearing aids were also found associated with tinnitus masker. A feedback on the results of association of mould with tinnitus masker from a professional audiologist of a large NHS (National Health Services, UK) was also taken to better understand them. The results were obtained with different accuracy for different techniques. For example, the chi-squared test results were obtained with 95\% accuracy, for Support and Confidence only those results were retained which had more than 1\% Support and $80 \%$ Confidence.

Conclusions: The variables audiograms, age, gender, hearing aid type and mould were found associated with the choice of tinnitus masker in the literature and by using statistical and data mining techniques. The further work in this research would lead to the development of a decision support system for tinnitus masker with an explanation that how that decision was obtained.

Keywords: Tinnitus; Masker; Audiogram; Age; Gender; ITE (in the ear)/BTE (behind the ear) hearing aids; Mould

\section{Background and significance}

This research deals with a big heterogeneous audiology data from a large NHS (National Health Services) facility, containing audiograms, structured data (such as age, gender, diagnosis) and free text (containing specific information about each patient, i.e., clinical comments). This research looks for factors associated with tinnitus

Correspondence: Naveed.Anwar@open.ac.uk

The Open University, Milton Keynes, UK masker on the basis of large data by doing the mining and analysis of this data.

Deafness Research UK (2010) states that nearly five million people in the UK live with tinnitus. Some estimates show that 40 million people in the United States may be affected by tinnitus and 10 million of them consider their problem significant (Folmer 2002). In many cases the exact reason for tinnitus is not known and diagnosis typically begins with a visit to an ear specialist. A detailed clinical evaluation, including a thorough patient history and physical check-up of the head and neck 
together with the different nerves in that region, and audiometric testing are performed by the doctor for the diagnosis of tinnitus and its causes. For example, ear wax, ear infection, and vestibulocochlear nerve (auditory nerve) that controls the sense of hearing and balance are checked. A detailed audiogram is also taken. Some tinnitus may be caused by neurologic, metabolic, or psychogenic disorders (Crummer and Hassan 2004). Depending on the type of tinnitus, either a special audiogram known as an auditory brainstem response (ABR) (a test that gives information about the inner ear (cochlea) and brain pathways for hearing) or a brain scan such as a magnetic resonance imaging (MRI) scan or computerised tomography (CT) may also be taken. In a few cases, blood pressure and possibly blood tests for hyperthyroidism (a condition that the thyroid gland makes too much thyroid hormone) are also performed. In a very few cases, a spinal tap procedure is used to find the fluid pressure in the skull and spinal cord. In the section below, some of the causes and problems associated with tinnitus and its treatment found in previous audiology research are described.

\section{Tinnitus causes}

Tinnitus can be caused by different factors including exposure to loud noises, head trauma, and a variety of diseases (Snow 2004). Tinnitus can be induced in $94 \%$ of the population by a few minutes of sound deprivation (Heller and Bergman 1953). Tinnitus can cause severe emotional distress to patients (Andersson and McKenna 1998), and results in disturbing the life of a person. Below are some of the causes of tinnitus found in the audiology literature:

\section{Hearing impairment}

Tinnitus is found to be highly correlated with hearing impairment and severity of impairment (Davis 1989). It has also been found that high frequency hearing loss is a predictor of tinnitus (Jakes and Stephens 1987). In the given audiology data, it was found that patients with mild to moderate hearing loss group were more likely to have tinnitus and be using a masker, and also in this group patients were more concerned with tinnitus than the hearing loss.

\section{Age}

The likelihood of having tinnitus increases with age and about $30 \%$ of the population affected with tinnitus have been found to be over 55 years of age (Lockwood et al. 1998). In a previous research on a large audiology data, it was found that patients having age less than or equal to 54 (i.e., Age $\leq 54$ ) were less likely to have tinnitus (Anwar et al. 2011).

\section{Gender}

It is reported that women with tinnitus seem to report more psychological distress, though the exact cause of this gender difference is not well understood (Dineen et al. 1997). In a study on tinnitus and hearing loss in Sweden, Brunnberg et al. (2008) found a gender difference in hard-of-hearing 15-16-year-old young students, but they did not find the gender difference in students with normal-hearing and having tinnitus. In a previous study on a large audiology data, there was no association found of gender with tinnitus (Anwar et al. 2011). Miekle and Griest (1989) found that more male tinnitus sufferers had histories of noise exposure than women, and that women have a greater tendency to report tinnitus on both sides (as opposed to in one ear only) than men.

\section{Tinnitus treatments and problems associated with them}

Jastreboff and Hazell (2004) described tinnitus treatment a promising, expensive and complex that may span from several months to a couple of years. It is described in the literature that there is neither any cure for tinnitus (Vio and Holme 2005; Gander et al. 2011) nor any licensed medications for minimising its symptoms (Vio and Holme 2005). For example, there is no Food and Drug Administration (FDA) or European approved drug specifically for treating subjective tinnitus (Langguth et al. 2009). The reason for this is the heterogeneity observed in the tinnitus population (Moller 1997; Landgrebe et al. 2010), so there is no single theory, model or hypothesis that explains the presence of tinnitus in all those affected (Baguley and McFerran 2002). Gander et al. (2011) mentioned that there are no comprehensive surveys about tinnitus referral systems from the clinician's perspective. Thus, most of the tinnitus treatment options are mainly for lessening or managing the existing symptoms of tinnitus by making them less distressing (Jastreboff and Hazell 2004; Vio and Holme 2005). Landgrebe et al. (2010) mentioned that one of the major problems of tinnitus research is that it is a subjective experience (like pain) and cannot be measured by using objective measurement methods. For these reasons, it is difficult to predict who will suffer from tinnitus (Tyler and Stouffer 1989). Tinnitus can only be quantified by using self-rating questionnaires. It is noted that an important factor for effective management of tinnitus patients is a quick process of triage and referral to an appropriate professional (Gander et al. 2011).

The fitting of masking devices plays an important part in the management of the many different forms of tinnitus (Hazell et al. 1985; Henry et al. 2004). Tinnitus maskers are small devices worn like hearing aids. They give relief from tinnitus by giving out a "white noise" or an ultra-sonic signal to cover up the internal sounds heard. Tinnitus masking has been in use as a clinical 
technique since 1976 (Vernon 1976). There are no harmful effects of masking on hearing (Hazell et al. 1985). The other treatments that are useful in the management of tinnitus are as follows:

- Hearing aids are found to give masking relief for about $12 \%$ of tinnitus patients (Vernon 1988). But in a recent study it was found that tinnitus patients with hearing aids report slightly fewer benefits and more problems with their hearing aids particularly for background sounds and intolerance of sounds (Andersson et al. 2011).

- On some occasions, when none of the other treatments is effective for tinnitus, surgery is advised to produce relief from severe effects of tinnitus. These cases are because of injuries in the inner ear and are generally related with severe or total deafness. These surgical procedures require a high degree of technical skill.

- TRT (Tinnitus Retraining Therapy) is another method of tinnitus treatment (Jastreboff and Jastreboff 2000). Its primary objective is to facilitate habituation to tinnitus (Henry et al. 2002; 2004). This method was first described in 1990, and is based on a neurophysiological model of tinnitus (Jastreboff 1990). It combines medical evaluation, counselling and sound therapy for the management of tinnitus to help the majority of tinnitus patients (Zhang et al. 2011).

- Tinnitus counselling is designed to enable the understanding of tinnitus to deal with it efficiently. It is usually conducted by hearing therapists. It is a discussion therapy that helps the patient to understand in detail about tinnitus and the methods to cope with it. Discussing tinnitus makes one understand more about it and possibly lessens its effect.

- Cognitive behavioural therapy (CBT) is related to many therapies that aim to deal with issues such as nervousness, sadness and post-traumatic stress disorder (PTSD). In the daily lives of people emotions affect their behaviour, and this is the basis of CBT. For example, a patient having a lack of information about tinnitus might have ideas that would make him more nervous and upset. But in reality these ideas are false and giving the correct information about tinnitus might help to improve the condition.

- Self-help techniques, such as taking rest, listening to music and joining tinnitus support groups, are also important for people for dealing with their tinnitus. For example, anxiety can increase tinnitus, therefore doing exercise regularly such as meditation or yoga might reduce tinnitus. Joining a tinnitus support group also gives the opportunity to share tinnitus experience with other tinnitus sufferers, which can also prove helpful by giving more understanding of tinnitus and how to better deal with it.

The minimal masking level (MML) of tinnitus is a possible predictor of acceptance of tinnitus masker treatment and also a measure of treatment success (Vernon et al. 1990; Jastreboff et al. 1994). The Beck Depression Inventory (BDI) is also used as a measure of depression (Beck et al. 1961). It uses a self-report instrument to measure the level of depression in tinnitus patients (Andersson and McKenna 1998; Kirsch et al. 1989). The higher the total scores of the responses, the greater the signs of depression.

\section{Tinnitus maskers}

One interesting and strange distinction among tinnitus patients regarding the effect of background noise for treating the tinnitus is that noise makes tinnitus worse for some patients but reduces tinnitus for others (Stouffer and Tyler 1990; Tyler and Baker 1983). It is important to provide careful counselling and follow up in order to obtain optimal results, when fitting a device, masker, aid or combination instrument (Hazell and Wood 1981). Maskers are often more effective than hearing aids (Hazell et al. 1985), though hearing aids have been reported to provide masking relief for about $12 \%$ of tinnitus clinic patients (Vernon 1988). Opinion about the effectiveness of maskers in the management of tinnitus varies as $71-88 \%$ of masker users and 53-68\% of hearing aid users found their instrument helpful when tinnitus was troublesome (Coles and Hallam 1987). A study on a group of tinnitus patients found that in the long term $45 \%$ of tinnitus patients used maskers with or without hearing aids, and 35\% used hearing aids alone (Stephens and Corcoran 1985). Another study performed initial investigations on the effectiveness of various sounds which were composed to provide tinnitus relief as compared to broadband or spectrally shaped noise, the two bands of noise commonly used in clinics, and it was found that those specially designed sounds were more effective than the typically used sounds (Henry et al. 2004). Masking for speech tests commonly uses white noise (actually broadband noise), pink noise, speech-shaped noise, or multitalker babble (Gelfand 2009). Broadband noise contains a wide range of frequencies, i.e. upwards of several octaves. White noise is a broadband noise which offers equal energy in all frequencies. Pink noise features less energy in the higher frequencies (above $8000 \mathrm{~Hz}$ ) and is the noise that most mimic day to day living. Speech shaped noise has a spectrum that approximates of the long-term spectrum of speech. Multitalker babble is made by recording the voices of many people who are talking simultaneously, resulting in an unintelligible babble. 
The choice of different masking devices in various studies is rarely specified as this is often based on their local availability and the clinician's link with the different manufacturers rather than on any scientific appraisal (Hazell et al. 1985). There are two types of masking: continuous (subdivided into complete and partial masking) and inhibitory. In continuous complete masking, the noise "covers up" the tinnitus so that it is not audible while the masker is in use (Coles and Hallam 1987; Newman and Sandridge 2006). Its applicability depends on the noise having a more acceptable quality then the tinnitus. The complete masking phase is only recommended for patients requiring immediate relief (Newman and Sandridge 2006). For all other patients, partial masking is the recommended initial phase for sound therapy. In partial masking, a masker is used to deliver such a lowlevel background sound at level a slightly below the tinnitus, which facilitates tinnitus habituation (Newman and Sandridge 2006). Inhibitory masking is an alternative to continuous masking, as its frequency-specificity is much greater than for continuous masking (Coles and Hallam 1987). In this research, the audiology database has the records of different types of maskers prescribed to patients, and it was found that patients classified in moderate to severe hearing loss group were less likely to have tinnitus masker which is described later in the section of "Clusters of audiology data for tinnitus masker".

\section{Problems with Tinnitus studies-small samples of tinnitus data}

Landgrebe et al. (2010) mentioned that many clinical studies of tinnitus have used small patient samples, which makes it difficult to find the forecasters of treatment response for the various methods. They further stated that there is limited inter-study comparison due to various procedures used for the assessment of tinnitus and their varying outcome elements. Because of these reasons there is a need for a global repository of tinnitus patients who have undertaken certain treatments, and are assessed during the course of this treatment with standardised instruments such as, psychoacoustic measures and questionnaires. This repository has been constructed by the Tinnitus Research Initiative (TRI) database project (Tinnitus Research Initiative, 2013), which is also discussed in the paper by Landgrebe et al. (2010). The main purposes of this database are: to collect a standardised set of data on patient characteristics, treatments, and outcomes from tinnitus patients consulting specialized tinnitus clinics all over the world (currently 19 centres in 11 countries), outlining various subtypes of tinnitus from the data that has been collected at these centres, and discovering forecasters for individual treatment response based on the clinical profile. This database started in 2008 and now it contains the data of almost 3000 patients. It is anticipated that the number of centres will increase and this will grow the patient data quickly, so that this global database will help the research in future and will add to the expansion of patients' treatment on the basis of facts.

Another repository, which in fact was the first attempt at systematically collecting tinnitus patient related data and making it freely available was the web-based Oregon Tinnitus Data Archive (Meikle 1997) http:// www.tinnitusarchive.org/ (Accessed 7 November 2013). This database contains a statistical overview of past and present status of patients' tinnitus data (such as patients' age as a frequency histogram, comparison of the mean audiograms for different groups of patients), various outcomes of tinnitus examinations (such as, sound frequency equal to the perceived pitch of a patient's tinnitus), audiological examinations (such as, hearing levels), and demographic information for 1630 patients with tinnitus who were examined at Tinnitus Clinic of the Oregon Hearing Research Center, Department of Otolaryngology, Oregon Health \& Science University, between 1981 to 1994. This database was created for classifying and preparing different studies of tinnitus causes, for research on the tinnitus affected population such as assessing and managing tinnitus. The existence of this database has contributed much towards the understanding of tinnitus (Folmer and Griest 2003) but there are limitations to it (Landgrebe et al. 2010), such as it contains only cross-sectional data, i.e., does not inform about the relation between specific tinnitus features and the outcomes of specific treatment methods for tinnitus. This database has highly selected patient data and does not contain any validated measures for the severity of tinnitus. This database was last updated in 1994.

This research is based on a large data from a hearing aid clinic. It contains 180,000 individual records covering more than 23,000 different patients. This data is unique in that it has both types of hearing aids that is BTE (behind the ear) and ITE (in the ear) prescribed to patients as ITE hearing aids are generally not available on NHS, UK. The factors found in the literature for tinnitus masker are audiograms, age, gender and hearing aid type. These factors along with other factors-mould and comment texts, are also available in the available audiology data. All the available records in the database for each field (factors mentioned above) under study were

\begin{tabular}{|c|c|c|c|c|c|c|}
\hline & ac250 & ac500 & ac1K & ac2K & ac4K & ac8K \\
\hline $\mathrm{C} 1$ & 62.34 & 62.57 & 63.55 & 67.34 & 77.57 & 83.50 \\
\hline C2 & 21.17 & 17.66 & 17.09 & 20.27 & 34.57 & 42.50 \\
\hline
\end{tabular}


Table 2 Class exemplars of each cluster of right ear air conduction frequencies for comments text

\begin{tabular}{lllllll}
\hline & ac250 & ac500 & ac1K & ac2K & ac4K & ac8K \\
\hline C1 & 73.66 & 73.00 & 74.99 & 80.48 & 91.08 & 108.21 \\
C2 & 35.17 & 33.56 & 35.87 & 43.26 & 55.90 & 66.50 \\
\hline
\end{tabular}

used for tinnitus with/without masker, keeping the criterion that none of the field values should be empty. Although, this research uses a large audiology data but it also faced the problem of limited data for tinnitus, as there were only 1,316 records for tinnitus and other diagnosis.

\section{Objective}

The objective of this research is to find the factors associated with tinnitus masker from the literature, and by using the large amount of audiology data available from a large NHS, UK hearing aid clinic.

\section{Materials and methods}

This large audiology data contains two types of data about tinnitus that is, one of the diagnosis types given in the diagnosis field is "tinnitus", and there is also a "masker" field where the type of tinnitus masker prescribed (if any) is recorded, in this study for processing the data for masker field only the presence or absence of masker is considered. It is important to mention that this audiology data was anonymised as there were $\mathrm{NO}$ actual patient ids, names, addresses and phone numbers given. Thus, no actual patient can be traced down.

\section{Clusters of audiology data for tinnitus masker}

The background and significance section discussed a number of causes and problems related to tinnitus and maskers. Tinnitus causes such as hearing impairment, age and gender have been found in the literature and these factors will also be used in this study. The most common treatment for tinnitus is the use of a masker. Since this large database includes both which patients were diagnosed with tinnitus and which were fitted with a tinnitus

Table 3 Observed and expected frequencies for right ear tinnitus with/without masker

\begin{tabular}{llll}
\hline Tinnitus & Cluster $\mathbf{1}$ & Cluster 2 & Row total \\
\hline With masker & 40 & 391 & 431 \\
& $(65.58)$ & $(365.42)$ & \\
& {$[654.27]$} & {$[654.27]$} & \\
Without masker & 133 & 573 & 706 \\
& $(107.42)$ & $(598.58)$ & \\
& {$[654.27]$} & {$[654.27]$} & \\
Column Total & 173 & 964 & 1137 \\
\hline
\end{tabular}

Table 4 Overall $\chi^{2}$

\begin{tabular}{llll}
\hline Fields & Overall $\boldsymbol{X}^{2}$ & $\begin{array}{l}\text { Degrees of } \\
\text { freedom }(\mathbf{d f})\end{array}$ & $\mathbf{P}$ \\
\hline Tinnitus with or without masker & 18.95 & 1 & $\mathrm{P}<0.001$ \\
Comments text & 4243.87 & 668 & $\mathrm{P}<0.001$ \\
\hline
\end{tabular}

masker, these fields can act as the gold standard for finding significant variables associated with tinnitus masker.

Clustering of hearing aid patient audiograms was performed on right ear air conduction (ac) frequencies from 250 to $8000 \mathrm{~Hz}$ (given in Tables 1 and 2) by using the k-means algorithm on 10,437 and 1,316 records with comments text and diagnosis (tinnitus and Others) respectively. The two main clusters were found for two sets of records, those for which the diagnosis field was filled in, and those for which the comment text field was filled. In each case the class exemplar (cluster centroid) of each cluster, being the mean of the audiograms contained within each cluster, was calculated (including only tinnitus diagnosis), as shown in Tables 1 and 2. In Table 1, cluster 1 (or C1) corresponds to "moderate to severe hearing loss" and cluster 2 (or C2) corresponds to "normal or near-normal hearing" for tinnitus with or without a masker. In Table 2, the class exemplars show that cluster 1 (or C1) consists of patients with "severe hearing loss" and cluster 2 (or C2) consists of patients with a "mild to moderate hearing loss" for comment text/clinical comments.

The Chi-squared test is a simple way to provide estimates of quantities of interest and related confidence intervals (Altman 1991). It is a measure of associations between variables (such as the fields of the tables in a relational database) where the variables are nominal and related to each other (Lucy 2005). The Chi-squared test is popular in the medical domain because of its simplicity. It has been used in pharmacology to classify text according to subtopics (Oakes et al. 2001). The resulting chi-squared value is a measure of the differences between a set of observed and expected frequencies within a population, and is given by the formula:

$$
X^{2}=\sum_{i=1}^{r} \sum_{j=1}^{c} \frac{\left(O_{i j}-E_{i j}\right)^{2}}{E_{i j}}
$$

where $r$ is the number of unique terms in a particular field of the patient records such as Tinnitus with/

Table 5 Clusters for the records with tinnitus with or without masker field with positive and negative keywords

\begin{tabular}{lll}
\hline & Positive keywords & Negative keywords \\
\hline C1 & Not found & Tinnitus with masker \\
C2 & Not found & Not found \\
\hline
\end{tabular}


Table 6 Clusters for the records with text field (comments text) with positive and negative keywords

\begin{tabular}{lll}
\hline \multicolumn{2}{c}{ Positive keywords } & Negative keywords \\
\hline C1 & $\begin{array}{l}\text { audio, mould, be34, be52, be36, unmask, be54, sil, ref, tsa, gp, ca, } \\
\text { OTHERS, rt, suitabl, be201 }\end{array}$ & $\begin{array}{l}\text { masker, rev, tinnitu, appt, fta, help, review, aid, further, nfa, progress, } \\
\text { 2000, ok, canc, counsel, cope, 2001, dna }\end{array}$ \\
C2 & masker, rev, tinnitu, appt, fta, help, review, aid, further, nfa & audio, mould, be34, be52, be36, unmask, be54, sil, ref
\end{tabular}

without masker, corresponding to rows in Table 3. c is the number of categories in the data (such as clusters) corresponding to columns in Table 3.

Table 3 is the table produced for tinnitus with masker occurring in the masker field where cluster 1 represents "moderate to severe hearing loss" and cluster 2 represents "normal or near-normal hearing". In Table 3, Observed frequencies appear at the top of each cell, Expected frequencies are in ( ), and (Observed frequency - Expected frequency) $)^{2}$ values are shown in [ ]. For example, if 40 of the masker fields of the records of patients in cluster 1 contained "masker", a value of 40 was recorded for masker being associated with that cluster. These values were the "observed" values, denoted $\mathrm{O}_{\mathrm{ij}}$ in the formula above. The corresponding "expected" values $\mathrm{E}_{\mathrm{ij}}$ were found by the formula:

$$
\text { Row total } \times \text { Column total/Grand Total }
$$

The row total for "tinnitus with masker" is the total number of times "masker" was prescribed to patients in the two clusters $=40+391=431$. The column total for cluster 1 is the total number of patients assigned to cluster 1 over all tinnitus patients $=173$. The grand total is the total number of patient records in the study $=1137$. Thus the "expected" (in this case $\mathrm{E}_{\mathrm{ij}}=\mathrm{E}_{11}$ ) number of "tinnitus with masker' in cluster 1 was $431 * 173 / 1137=65.58$. The significance of this is that the observed value is less than the expected value, suggesting that there is a negative degree of association between the "tinnitus with masker" and the severe hearing loss cluster. The remainder of the test is then performed to discover if this association is statistically significant. Next the $\mathrm{O}_{\mathrm{ij}}$ and $\mathrm{E}_{\mathrm{ij}}$ values were used to calculate an overall chi-squared value for the relationship between "tinnitus with masker" and cluster, using the Eq. 1 in Table 4. From this data it could be shown with 99.9\% confidence, that these keywords were not randomly

Table 7 Overall $\chi^{2}$ with tinnitus with/without masker

\begin{tabular}{llll}
\hline Fields & Overall $\boldsymbol{X}^{\mathbf{2}}$ & $\begin{array}{l}\text { Degrees of } \\
\text { freedom }(\mathbf{d f})\end{array}$ & $\mathbf{P}$ \\
\hline Age & 11.31 & 1 & $\mathrm{P}<0.001$ \\
Comments text & 416.49 & 83 & $\mathrm{P}<0.001$ \\
Gender & 0.02 & 1 & $\mathrm{P}=0.8875$ \\
BTE/ITE aid & 17.16 & 1 & $\mathrm{P}<0.001$ \\
Mould & 93.16 & 2 & $\mathrm{P}<0.001$ \\
\hline
\end{tabular}

distributed, and that some keywords definitely are more associated with some clusters.

The words most typical and atypical of each cluster are shown in Tables 5 to 6 . These automatically discovered words provided a suitable set of both positive and negative labels for each of the clusters. The labels seem intuitively reasonable. For example, in Table 5, "tinnitus with masker" is found to be atypical of moderate to severe hearing loss cluster, means patients having moderate to severe hearing loss were not using tinnitus masker. This is a common finding in audiology clinics that for severe hearing loss patients, masker might be of no use because they are unable to hear it. In Table 6, it appeared that the patients in cluster 2 , the mild to moderate hearing loss group, were more concerned about tinnitus (ringing in the ears) than hearing loss. Thus the words tinnitus and masker (a device using the sound to provide an immediate sense of palliative relief from tinnitus) were typical of this cluster and also are atypical of cluster 1, the severe

Table 8 Categories with positive and negative keywords in records with a tinnitus with/without masker

\begin{tabular}{|c|c|c|}
\hline & Positive keywords & Negative keywords \\
\hline \multirow[t]{2}{*}{ Age $<=55$} & * & * \\
\hline & Not found & Not found \\
\hline \multirow[t]{2}{*}{ Age $>55$} & * & * \\
\hline & Not found & Not found \\
\hline \multirow[t]{6}{*}{ Tinnitus without masker } & $* *$ & $* *$ \\
\hline & counsel, tinnitu & Not found \\
\hline & $* * *$ & $* * *$ \\
\hline & Not found & Not found \\
\hline & $* * * *$ & $* * * *$ \\
\hline & 2107 & N8 \\
\hline \multirow[t]{6}{*}{ Tinnitus with masker } & $* *$ & $* *$ \\
\hline & masker & counsel, tinnitu \\
\hline & $* * *$ & $* * *$ \\
\hline & Not found & BTE \\
\hline & $* * * *$ & $* * * *$ \\
\hline & N8 & 2107 \\
\hline \multirow[t]{2}{*}{ Male } & * & * \\
\hline & Not found & Not found \\
\hline \multirow[t]{2}{*}{ Female } & $*$ & * \\
\hline & Not found & Not found \\
\hline
\end{tabular}


Table 9 Yule's Q for tinnitus with/without masker and mild-to-moderate hearing loss cluster/normal or near-normal hearing loss cluster

\begin{tabular}{llllll}
\hline Tinnitus & $\begin{array}{l}\text { Severe hearing } \\
\text { loss cluster (P) }\end{array}$ & $\begin{array}{l}\text { Normal or near-normal } \\
\text { hearing loss cluster (P) }\end{array}$ & $\begin{array}{l}\text { Severe hearing } \\
\text { loss cluster (A) }\end{array}$ & $\begin{array}{l}\text { Normal or near-normal } \\
\text { hearing loss cluster (A) }\end{array}$ & $\begin{array}{c}\text { Yule's Q } \\
\text { With masker }\end{array}$ \\
Without masker & 13 & 391 & 133 & 573 & -0.39 \\
\hline
\end{tabular}

hearing loss group. Similarly, in (Table 6) cluster 1, the atypical words "canc" (cancelled) and "dna" (did not attend) show that patients with severe hearing loss were less likely to cancel (or simply fail to attend) their appointments. "Tinnitus" appears as "tinnitu" and "Suitable" appears as "suitabl" in Table 6, since all the text was passed through Porter's (Porter 1980) stemmer for the removal of grammatical endings.

\section{Chi-squared and associations in tabular audiology data for tinnitus masker}

The Chi-squared test can show the existence of associations in the data, and other association measures based on the contingency table can be used to measure the strength of the relationship between the variables in medical data. Support and confidence are measures of the interestingness of associations between variables (Ordonez et al. 2006; Bramer 2007). They show the usefulness and certainty of discovered associations. Strong associations are not always interesting, because support and confidence do not filter out uninteresting associations (Han and Kamber 2006). Thus, support and confidence in the medical domain should be augmented by chi-squared $\left(\chi^{2}\right)$.

Discovery of associations with the Chi-squared test tables The associations of each of the variables (age, comments text, gender, ITE/BTE aid and mould) with "tinnitus with masker" are shown in Table 7 with their overall chi-squared values. In Table 8, typical and atypical keywords found for tinnitus masker associated with variables mentioned above are given, where $* * *, * * *$ and $* * * *$ denote a "tinnitus masker", "comments text", "ITE/BTE hearing aid" and "mould" categories respectively. For example, in Table 8 keywords "tinnitu" for "tinnitus" and "counsel" were found associated with tinnitus without masker that is, tinnitus suffering patients who are NOT using masker were counselled on tinnitus. Mould "N8"

Table 10 Yule's Q for ITE/BTE aid and tinnitus with/without masker

\begin{tabular}{llllll}
\hline $\begin{array}{l}\text { Hearing } \\
\text { aid type }\end{array}$ & $\begin{array}{l}\text { With } \\
\text { masker }(\mathbf{P})\end{array}$ & $\begin{array}{l}\text { Without } \\
\text { masker }(\mathbf{P})\end{array}$ & $\begin{array}{l}\text { With } \\
\text { masker }(\mathrm{A})\end{array}$ & $\begin{array}{l}\text { Without } \\
\text { masker }(\mathbf{A})\end{array}$ & Yule's Q \\
\hline ITE & 67 & 101 & 15 & 82 & 0.57 \\
BTE & 15 & 82 & 67 & 101 & -0.57 \\
\hline
\end{tabular}

was found associated with tinnitus masker and mould "2107" for people with tinnitus but not using masker. It is important to mention that BTE aids were found atypical with tinnitus masker.

An audiologist from a large NHS facility said that the "N8" mould is an open fitting ear mould which doesn't obstruct the ear canal, so allows natural hearing to complement output from the hearing aid and is required when there is a small or no hearing loss, for example, in tinnitus maskers. Patients with significant hearing loss with tinnitus are generally given hearing aids and the environmental sounds picked up will mask the tinnitus. Hence there is a high correlation between "N8" mould and tinnitus masker.

\section{Measures of association (Yule's Q) in categorical audiology data for tinnitus masker}

The Chi-squared test informed about the presence of an association, while Yule's $Q$ produces the strength and direction of the association. Yule's $Q$ is in the range -1 to +1 , where the sign indicates the direction of the relationship and the absolute value indicates the strength of the relationship. In Tables 9, 10, 11, 12, Yule's Q values for tinnitus with/without masker and severe hearing loss cluster/mild-to-moderate hearing loss cluster, hearing aid type, age and gender are given. The symbols " $(\mathrm{P})$ " and "(A)" in tables, stand for present and absent. In Table 10, a Yule's $Q$ value of 0.57 shows that there is a positive association between the ITE aid and tinnitus with masker, which could be related with the results found in Table 8 above, that BTE aids were atypical of tinnitus with masker. In Table 11, there was a weak positive association of "tinnitus with masker" with Age $<=55$, meaning that patients of Age $<=55$ having tinnitus tended to use a masker, while patients of Age $>55$ having tinnitus tended not to use a masker.

Table 11 Yule's Q for tinnitus with/without masker and age

\begin{tabular}{llllll}
\hline Tinnitus & $\begin{array}{l}\text { Age }<=55 \\
\text { (P) }\end{array}$ & $\begin{array}{l}\text { Age }>\text { 55 } \\
\text { (P) }\end{array}$ & $\begin{array}{l}\text { Age }<=55 \\
\text { (A) }\end{array}$ & $\begin{array}{l}\text { Age }>\mathbf{5 5} \\
\text { (A) }\end{array}$ & Yule's Q \\
\hline With masker & 240 & 182 & 328 & 377 & 0.20 \\
$\begin{array}{l}\text { Without } \\
\text { masker }\end{array}$ & 328 & 377 & 240 & 182 & -0.20 \\
\hline
\end{tabular}


Table 12 Yule's Q for tinnitus with/without masker and gender

\begin{tabular}{llllll}
\hline Tinnitus & Male (P) & Female (P) & Male (A) & Female (A) & Yule's Q \\
\hline With masker & 246 & 206 & 396 & 337 & 0.01 \\
$\begin{array}{l}\text { Without } \\
\text { masker }\end{array}$ & 396 & 337 & 246 & 206 & -0.01 \\
\hline
\end{tabular}

Support and confidence for tinnitus masker associations Support and confidence are measures of the interestingness of a rule. Support reflects the usefulness of a rule, and confidence its certainty (Han and Kamber 2006). To find the significant associations, the criteria that support is more than or equal to a minimum threshold values called minsup, which is typically 0.01 (or 1\%) and also confidence is more than or equal to a minimum threshold value called minconf, which is typically 0.8 (or $80 \%$ ) are used (Bramer 2007). So, in Tables 13, 14, 15 the only rule that meet such criteria is tinnitus masker associated with ITE aid in Table 13. The Tables 14 and 15 do not meet this criterion. For example, the overall chi-squared value 17.16 gave $99 \%$ confidence that there is indeed an association between tinnitus with/without masker and BTE/ITE aid (as shown in Table 7). The Yule's Q value of 0.57 for ITE hearing aid and tinnitus with masker (in Table 10) shows that this association is both positive and strong. Rule's support and confidence values of 0.25 and 0.82 (in Table 13) show this association is only moderately useful, but highly certain.

\section{Conclusion to tinnitus and maskers}

In this research the literature survey to find the factors associated with tinnitus and masker is done, and it also uses a number of statistical and data mining techniques for finding the significant factors associated with tinnitus masker. The first technique applied was k-means clustering on audiograms, which found two clusters for "tinnitus with or without masker" and "comments text". These clusters were also automatically assigned labels that is, keywords associated with each cluster are found, which helps in the interpretation of the cluster. For audiograms k-means clustering method was selected, as it works well with large data and produce quick results. The Chisquared test is used for the discovery of associations for

Table 13 Support and confidence for ITE/BTE aid and tinnitus with/without masker

\begin{tabular}{llllll}
\hline & \multicolumn{2}{l}{ Tinnitus with masker } & & \multicolumn{2}{l}{ Tinnitus without masker } \\
\cline { 2 - 3 } Hearing aid type & Support & Confidence & & Support & Confidence \\
\hline ITE & 0.25 & 0.82 & 0.38 & 0.55 \\
BTE & 0.06 & 0.18 & 0.31 & 0.45 \\
\hline
\end{tabular}

Table 14 Support and confidence for tinnitus with/without masker and age

\begin{tabular}{llllll}
\hline & \multicolumn{2}{l}{ Age $<=55$} & & Age $>\mathbf{5 5}$ & \\
\cline { 2 - 3 } Tinnitus & Support & Confidence & & Support & Confidence \\
\hline With masker & 0.21 & 0.42 & & 0.16 & 0.33 \\
Without masker & 0.29 & 0.58 & & 0.33 & 0.67 \\
\hline
\end{tabular}

free text comments, diagnosis, hearing aid type, age and gender. Then the measures of associations for the same factors are calculated using Yule's Q, support and confidence. In fact, significant associations in the heterogeneous audiology data are looked for, with the ultimate aim of looking for factors which patients would most benefit from being fitted with a tinnitus masker.

The clustering was performed on audiograms which produced two main clusters of audiograms. The associations of clusters of audiograms type with tinnitus were confirmed in the section of clustering, and also by literature review in the "Background and significance" section for tinnitus. Thus, it has been demonstrated that the audiogram is a factor influencing the prescription of a tinnitus masker.

In Table 16, the candidate variables associated with tinnitus masker that is "factors influencing the choice of tinnitus masker" and the reasons for choosing them are summarised. These results demonstrated that tinnitus maskers are more likely to be fit to individuals with milder forms of hearing loss, and the factors age, gender, type of hearing aid and mould were all found significantly associated with tinnitus masker. In particular, those patients having Age $<=55$ years were more likely to wear a tinnitus masker, as well as those with milder forms of hearing loss. ITE (in the ear) hearing aids were also found associated with tinnitus masker. These variables agree fairly well with those factors found in the literature (as given under the heading of "Tinnitus causes"). Thus, the variables were found in the literature and by using statistical and data mining techniques for tinnitus masker.

A feedback on the results of association of mould with tinnitus masker from a professional audiologist of a large NHS (National Health Services, UK) was also taken to better understand them. The results were obtained with

Table 15 Support and confidence for tinnitus with/without masker and gender

\begin{tabular}{llllll}
\hline & Male & & & Female & \\
\cline { 2 - 3 } \cline { 5 - 6 } Tinnitus & Support & Confidence & & Support & Confidence \\
\hline With masker & 0.21 & 0.38 & 0.17 & 0.38 \\
Without masker & 0.33 & 0.62 & 0.28 & 0.62 \\
\hline
\end{tabular}


Table 16 Candidate variables with their reason for association with research question: factors influencing the choice of tinnitus masker

\begin{tabular}{|c|c|}
\hline Variables found & Reason for choosing the variable(s) \\
\hline Audiograms & $\begin{array}{l}\text { Tinnitus maskers were found atypical with moderate to severe hearing loss cluster of audiograms in Table 5, } \\
\text { and Yule's Q found tinnitus masker associated with normal or near-normal hearing loss cluster of audiograms in Table } 9 .\end{array}$ \\
\hline Gender & Yule's Q found very weak positive association of males with tinnitus masker in Table 12. \\
\hline ITE/BTE aid & $\begin{array}{l}\text { Chi-squared test found BTE aids atypical of tinnitus maskers in Table } 8 \text {. By using Yule's Q, and Support and } \\
\text { Confidence tinnitus maskers were found associated with ITE aids in Table } 10 \text { and Table } 13 \text { respectively. }\end{array}$ \\
\hline Age & Yule's Q found weak positive association of tinnitus masker with Age < =55 in Table 11. \\
\hline Mould & Mould was found associated with tinnitus with masker using chi-squared test in Table 8. \\
\hline
\end{tabular}

different accuracy for different techniques. For example, the chi-squared test results were obtained with $95 \%$ accuracy, for Support and Confidence only those results were retained which had more than 1\% Support and $80 \%$ Confidence. It is important to mention that some other factors that could not be tested with this data were cosmetic reasons, comfort in wearing, ease of use with spectacles and the measure of masking relief provided by tinnitus maskers.

The sets of variables found in Table 16 will be carried forward for constructing a decision support system (DSS). In future, these variables can be combined into mathematical models which will form the basis of a DSS, where unseen patient records will be presented to the system and the associated probability that the patient should be fitted with a tinnitus masker as opposed to NO tinnitus masker will be returned. By constructing these models, it will be seen which variables contribute most in reaching a final decision for tinnitus masker.

\section{Study approval}

It is a study involving audiology data NOT human directly. This data was given to the Department of Computing, Engineering \& Technology, University of Sunderland, Sunderland, UK, for Research purpose by a large National Health Services facility after making the data anonymised. So, there was no need to obtain any permission as long as it is being used for research purpose. The author has been working on this data for his research at University of Sunderland and this paper is one of the outcomes of his research.

\section{Article summary}

\section{Article focus}

- A large database of audiology data from a large NHS (National Health Services, UK) facility

- Data analysis of audiology data using various statistical and data mining techniques

- Tinnitus and masker

\section{Key messages}

- Unconventional study to find factors associated with tinnitus masker

- Association analysis of tinnitus and masker

- Improved quality of service in audiology clinics, so that the variables found can be emphasized, and when the decision support system to predict for tinnitus masker would be developed

\section{Strengths and limitations of this study}

- This research includes both BTE (behind the ear) and ITE (in the ear) hearing aids, as ITE aids are generally not available on NHS (National Health Services) prescriptions

- This research finds variables associated with tinnitus masker

- This study includes comment and feedback given by a professional audiologist from a large NHS facility on the association of mould with tinnitus masker

- It does not include factors such as comfort in wearing of masker, ease of use with spectacles and measure of masking relief

\section{Competing interests}

The author declares that he has no competing interests.

\section{Acknowledgements}

The author would like to thank concerned people at a large NHS facility for making the large set of anonymised audiology records available to him. He would also like to thank the principal audiologist of this NHS facility, and Dr. Michael Oakes (University of Wolverhamton, UK) and the anonymised reviewers for giving their comments and feedback on his work.

Received: 30 May 2013 Accepted: 24 October 2013

Published: 8 November 2013

\section{References}

Altman DG (1991) Practical statistics for medical research. Chapman \& Hall Publishers, London, pp 241-248, 211, 271

Andersson G, McKenna L (1998) Tinnitus masking and depression. Int J Audiol 37:174-182

Andersson G, Keshishi A, Baguley DM (2011) Benefit from hearing aids in users with and without tinnitus. Audiol Med 9(2):73-78 
Anwar MN, Oakes MP, McGarry K (2011) Chi-squared, Yule's Q and likelihood ratios in tabular audiology data. In: Ao SL, Gelman L (eds) Electrical engineering and applied computing, vol 90. Springer Netherlands, Dordrecht, pp 376-465

Baguley DM, McFerran DJ (2002) Current perspectives on tinnitus. Arch Dis Child 86:141-143

Beck AT, Ward CH, Mendelson M, Mock J, Erbaugh J (1961) An inventory for measuring depression. Arch Gen Psychiatry 4:561-571

Bramer M (2007) Principles of data mining. Springer-Verlag, London, pp 187-218, 224-231, 239-253

Brunnberg E, Lindén-Boström M, Berglund M (2008) Tinnitus and hearing loss in 15-16-year-old students: mental health symptoms, substance use, and exposure in school. Int J Audiol 47:668-694

Coles RRA, Hallam RS (1987) Tinnitus and its management. Br Med Bull 43:983-998

Crummer RW, Hassan GA (2004) Diagnostic approach to tinnitus. Am Fam Physician 69(1):120-126

Davis AC (1989) The prevalence of hearing impairment and reported hearing disability among adults in Great Britain. Int J Epidemiol 18:911-917

Deafness Research UK (2010) Better treatment today, hope for tomorrow, 25 years of hearing research. Manor Creative Limited, Eastbourne, England, p 14

Dineen R, Doyle J, Bench J (1997) Managing tinnitus: a comparison of different approaches to tinnitus management training. Br J Audiol 31:331-344

Folmer RL (2002) Long-term reductions in tinnitus severity. BMC Ear Nose Throat Disord, http://www.biomedcentral.com/1472-6815/2/3. Accessed 7 November 2013.

Folmer RL, Griest SE (2003) Chronic tinnitus resulting from head or neck injuries. Laryngoscope 113:821-827

Gander PE, Hoare DJ, Collins L, Smith S, Hall DA (2011) Tinnitus referral pathways within the National Health Service in England: a survey of their perceived effectiveness among audiology staff. BMC Health Serv Res 11:162

Gelfand AS (2009) Essentials of audiology, 3rd edn. Thieme Medical Publishers, USA, pp 274-301

Han J, Kamber M (2006) Data mining: concepts and techniques, 2nd edn. Morgan Kaufmann Publishers, USA, pp 79-80, 227-272

Hazell JWP, Wood SM (1981) Tinnitus masking-a significant contribution to tinnitus management. Br J Audiol 15:223-231

Hazell JWP, Wood SM, Cooper HR, Stephens SDG, Corcoran AL, Coles RRA, Baskill $J$, Sheldrake JB (1985) A clinical study of tinnitus maskers. Br J Audiol 19:65-146

Heller MF, Bergman M (1953) Tinnitus aurium in normally hearing persons. Ann Otol Rhinol Laryngol 62:73-83

Henry JA, Jastreboff MM, Jastreboff PJ, Schechter MA, Fausti SA (2002) Assessment of patients for treatment with tinnitus retraining therapy. J Am Acad Audiol 13:523-544

Henry JA, Rheinsburg B, Zaugg T (2004) Comparison of custom sounds for achieving tinnitus relief. J Am Acad Audiol 15:585-598

Jakes S, Stephens SDG (1987) Multivariate analyses of tinnitus complaint and change in tinnitus complaint: a masker study. Br J Audiol 21:259-272

Jastreboff PJ (1990) Phantom auditory perception (tinnitus): mechanisms of generation and perception. Neurosci Res 8:221-254

Jastreboff PJ, Hazell JWP (2004) Tinnitus retraining therapy-implementing the neurophysiological model. Cambridge University Press, New York, USA

Jastreboff PJ, Jastreboff MM (2000) Tinnitus Retraining Therapy (TRT) as a method for treatment of tinnitus and hyperacusis patients. J Am Acad Audiol 11:162-177

Jastreboff PJ, Hazell JWP, Graham RL (1994) Neurophysiological model of tinnitus: dependence of the minimal masking level on treatment outcome. Hear Res 80:216-232

Kirsch CA, Blanchard EB, Parnes SM (1989) Psychological characteristics of individuals high and low in their ability to cope with tinnitus. Psychosom Med 51:209-217

Landgrebe M, Zeman F, Koller M, Eberl Y, Mohr M, Reiter J, Staudinger S, Hajak G, Langguth B (2010) The Tinnitus Research Initiative (TRI) database: a new approach for delineation of tinnitus subtypes and generation of predictors for treatment outcome. BMC Med Inform Decis Mak 10:42

Langguth B, Salvi R, Elgoyhen AB (2009) Emerging pharmacotherapy of tinnitus. Expert Opin Emerg Drugs 14:687-702

Lockwood AH, Salvi RJ, Coad ML, Towsley ML, Wack DS, Murphy BW (1998) The functional neuroanatomy of tinnitus: evidence for limbic system links and neural plasticity. Neurology 50:114-120

Lucy D (2005) Introduction to statistics for forensic scientists. John Wiley \& Sons, England, pp 45-52
Meikle MB (1997) Electronic access to tinnitus data: the Oregon Tinnitus Data Archive. Otolaryngol Head Neck Surg 117:698-700

Meikle MB, Griest SE (1989) Gender-based differences in characteristics of tinnitus. Hear J 42(11):68-76

Moller AR (1997) Similarities between chronic pain and tinnitus. Am J Otol 18:577-585

Newman CW, Sandridge SA (2006) Incorporating group and individual sessions into a tinnitus management clinic. In: Tyler RS (ed) Tinnitus treatment: clinical protocols, chapter 14. Thieme Medical Publishers, New York, USA

Oakes M, Gaizauskas R, Fowkes H et al (2001) Comparison between a method based on the chi-square test and a support vector machine for document classification. Proceedings of ACMSIGIR, New Orleans., pp 440-441

Ordonez C, Ezquerra N, Santana CA (2006) Constraining and summarizing association rules in medical data. In: Cercone $\mathrm{N}$ et al (eds) Knowledge and information systems. Springer, New York, pp 259-283

Porter MF (1980) An algorithm for suffix stripping. Program 14:130-137

Snow JB (2004) Tinnitus: theory and management. BC Decker, Canada

Stephens SDG, Corcoran AL (1985) A controlled study of tinnitus masking. Br J Audiol 19:159-167

Stouffer JL, Tyler RS (1990) Characterization of tinnitus by tinnitus patients. J Speech Hear Disord 55:439-453

Tinnitus Research Initiative (2013) http://database.tinnitusresearch.org/index.php. Accessed 7 November 2013

Tyler RS, Baker LJ (1983) Difficulties experienced by tinnitus sufferers. J Speech Hear Disord 48:150-154

Tyler RS, Stouffer JL (1989) A review of tinnitus loudness. Hear J 42:52-57

Vernon J (1976) The use of masking for relief of tinnitus. In: Silverstein H, Norrell $\mathrm{H}$ (eds) Neurological surgery of the ear, vol 2. Aesculapius Publishing, Birmingham, pp 104-118

Vernon JA (1988) Current use of masking for the relief of tinnitus. In: Kitahara M (ed) Tinnitus, pathophysiology and management. Tokyo, Igaku-Shoin, pp 96-106

Vernon J, Greist S, Press L (1990) Attributes of tinnitus and the acceptance of masking. Am J Otolaryngol 11:44-50

Vio MM, Holme RH (2005) Hearing loss and tinnitus: 250 million people and a US \$10 billion potential market. Drug Discov Today 10(19):1263-1265

Zhang X, Thompson P, Raś ZW, Jastreboff P (2011) Mining tinnitus data based on clustering and new temporal features. In: Biba M, Xhafa F (eds) Learning structure and schemas from documents, vol 375. Studies in computational intelligence. Springer-Verlag, Berlin Heidelberg, pp 227-246

doi:10.1186/2193-1801-2-595

Cite this article as: Anwar: Mining and analysis of audiology data to find significant factors associated with tinnitus masker. SpringerPlus 2013 2:595.

\section{Submit your manuscript to a SpringerOpen ${ }^{\circ}$ journal and benefit from:}

- Convenient online submission

- Rigorous peer review

- Immediate publication on acceptance

- Open access: articles freely available online

- High visibility within the field

- Retaining the copyright to your article

Submit your next manuscript at springeropen.com 